\title{
A panoramic view of cancer
}

\section{A par}

\section{the Pan-Cancer \\ effort has \\ generated an}

unprecedented

wealth of

comparative

analyses for

cancer biology
Genomics has already made great contributions to our understanding of cancer biology but, until now, has focused on characterizing individual cancer types. The Pan-Cancer Initiative of The Cancer Genome Atlas (TCGA) has now taken the next step - comparative genomic analyses across the 12 cancer types for which genomic data have so far been generated.

In a coordinately published set of papers in Nature, Nature Genetics and other journals, the Pan-Cancer group has analysed up to 5,000 individual cancers, including cancers of the breast, uterus, ovaries, lung, brain, head and neck, colon and rectum, bladder, kidney and blood. Owing to the large sample sizes, the analyses are impressively highly powered and provide a range of insights. A few of the studies that make up this collection are discussed here, and the full set of papers is available as an online Focus (http://www.nature. com/ng/focus/tcga/index.html).

Kandoth, McLellan and colleagues focused on point mutations and small insertions and deletions (indels) from 3,281 tumours across the 12 tumour types to identify 127 significantly mutated genes. These genes are involved in a wide range of cellular processes. In addition to familiar culprits, these analyses emphasize the emerging importance of splicing, metabolism, proteolysis and chromatin modification in cancer. Perhaps not unexpectedly, the average number of alterations in these genes varies across tumour types, with the highest number ( six) in uterine and lung cancers and the lowest number ( two) in breast, kidney and ovarian cancers and in acute myeloid leukaemia.
Taking the example of chromatin remodellers, insights into their importance in cancer had already emerged from individual cancer genome analyses. However, it becomes apparent from this new study that different remodellers are preferentially mutated in different cancer types. Another insight is that, whereas alterations that affect histone modifiers, phosphoinositide 3-kinase signalling and genome integrity tend to have effects in many cancer types, those that affect transcriptional regulators and transforming growth factor- $\beta$ (TGF $\beta$ ) and WNT- $\beta$-catenin signalling tend to be associated with individual types of cancer.

To explore the patterns of mutations in the significantly mutated genes the authors turned to cluster analysis and uncovered patterns that reflect the tissue provenance of the cancer. They also gained insights into mutual exclusivity and co-occurrence among significantly mutated genes; for example, in breast cancers, mutations in TP53 (which encodes p53) and cadherin $1(C D H 1)$ are not seen together.

Importantly, from a translational perspective, these authors also carried out survival analysis across cancer types. They identified several genes that are significantly associated with particularly poor prognosis, including TP53, DNA methyltransferase 3A (DNMT3A) and the BAP1 gene, which encodes a ubiquitin carboxy-terminal hydrolase. Their results also emphasize the importance of knowing the clonal architecture of tumours from individual patients for optimizing treatment.

Also focusing on the same 12 tumour types, Ciriello et al. describe an inverse relationship between the number of recurrent somatic copy-number alterations (SCNAs) and the number of point mutations. These authors identified 30 tumour subclasses that are mostly tissue independent, many of which are characterized by a set of potentially therapeutically actionable targets.

SCNAs were also the focus of Zack and colleagues. They found whole-genome duplications in $37 \%$ of cancers, which also had higher rates of other types of SCNAs, as well as a few key point mutations and indels, including in TP53. Close comparisons of chromosome-internal and telomeric SCNAs suggested that they might arise as a result of mechanistically different processes. Tying in analyses of the point mutations and indels that were the focus of Kandoth, McLellan and colleagues, these authors found that, of the 140 regions carrying recurrent SCNAs, 50 carried significantly mutated genes, but 102 did not include any previously known oncogenes or tumour suppressors.

Collectively, the Pan-Cancer effort has generated an unprecedented wealth of comparative analyses for cancer biology. However, this is only the beginning - the data, which are publicly available, will be scrutinized by the wider scientific community, and the consortium will continue to generate more data for additional cancer types. The ultimate goal remains the same - to augment our understanding of cancer biology for improved diagnostics, prognostics and therapy.

Magdalena Skipper Senior Editor, Nature This article originally appeared in Nature Rev. Genet. (http://dx.doi.org/10.1038/nrg3602).

ORIGINAL RESEARCH PAPERS Kandoth, C. et al. Mutational landscape and significance across 12 major cancer types. Nature 502, 333-339 (2013) | Zack, T. I. et al. Pan-cancer patterns of somatic copy number alteration. Nature Genet. 45, 1134-1140 (2013)|Ciriello, G. et al. Emerging landscape of oncogenic signatures across human cancers. Nature Genet. 45, 1127-1133 (2013)

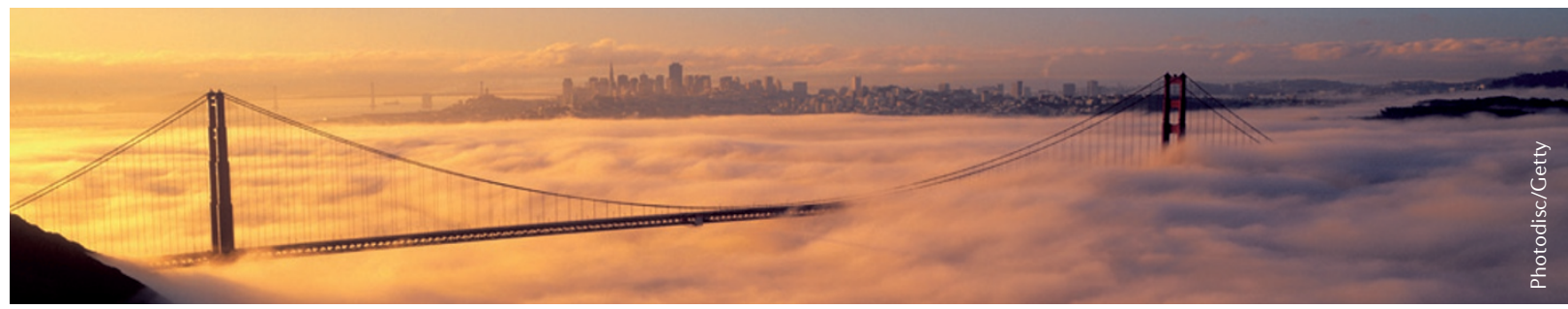

